\title{
LECTURE
}

\section{Pemphigus as a paradigm of autoimmunity and cell adhesion}

\author{
Masayuki Amagai \\ Department of Dermatology, School of Medicine, Keio University, Tokyo, Japan
}

(Received for publication on April 15, 2002)

\begin{abstract}
Pemphigus is a group of autoimmune blistering diseases of the skin and mucous membranes that are characterized histologically by intraepidermal blisters due to the loss of cell-cell adhesion of keratinocytes and immunopathologically by the finding of pathogenic IgG autoantibodies directed against the cell surface of keratinocytes. Identification of the target antigens has redefined pemphigus as an autoimmune disease against desmosomal cadherin or desmoglein. The IgG autoantibodymediated functional inhibition of desmoglein which plays an important role in the cell-cell adhesion of keratinocytes results in blister formation. Patients with pemphigus vulgaris and pemphigus foliaceus have IgG autoantibodies against desmoglein 3 and desmoglein1, respectively. Even complex clinical variations of pemphigus vulgaris and foliaceus are now logically explained by the desmoglein compensation theory. As an extension of this theory, the exfoliative toxin produced by Staphylococcus aureus, which causes staphylococcal scalded skin syndrome and bullous impetigo, was found to specifically cleave desmoglein1 and induce the identical histology to pemphigus foliaceus. Another recent innovation has been the development of an active disease mouse-model for pemphigus using autoantigen knockout mice, in which self-tolerance of the defective gene product is not acquired. When splenocytes from desmoglein 3 knockout mice are adoptively transferred into mice expressing desmoglein 3, anti-desmoglein3 IgG is stably produced in the recipient mice that develop the phenotype of pemphigus vulgaris. This model will be valuable not only for dissecting the cellular and molecular mechanisms in pathogenic antibody production but also for developing novel therapeutic strategies. (Keio J Med 51 (3): 133-139, September 2002)
\end{abstract}

Key words: cadherin, autoantibody, mouse model, desmoglein

\section{Pemphigus as an Autoimmune Disease Against Desmosomal Cadherin}

The term pemphigus stems from the Greek pemphix meaning blister or bubble and describes a group of chronic blistering skin diseases in which autoantibodies are directed against the cell surface of keratinocytes, resulting in the loss of cell-cell adhesion of keratinocytes through a process called acantholysis. ${ }^{1}$ Pemphigus can be divided into three major forms; pemphigus vulgaris, pemphigus foliaceus, and paraneoplastic pemphigus (Table 1).

In pemphigus vulgaris, essentially all patients have mucosal membrane erosions and more than half of them also have skin blisters and erosions. The blisters of pemphigus vulgaris develop in the deeper part of the epidermis, just above the basal cell layer. In pemphigus foliaceus, patients have only cutaneous involvement without mucosal lesions and the splits occur in the superficial part of the epidermis, mostly at the granular layer. Pemphigus vegetans is a vegetative variant of pemphigus vulgaris, and pemphigus erythematosus and fogo selvagem are localized and endemic variants of pemphigus erythematosus, respectively. Paraneoplastic pemphigus was more recently recognized as a disease distinct from the classic forms of pemphigus. ${ }^{2}$ Patients with paraneoplastic pemphigus have a known or occult associated neoplasm, usually of lymphoid tissue. Painful severe oral and conjunctival erosions are a prominent feature of paraneoplastic pemphigus.

The hallmark of pemphigus is the finding of IgG autoantibodies against the cell surface of keratinocytes. ${ }^{3}$ The pemphigus autoantibodies found in patients' sera play a primary pathogenic role in inducing the loss of 
Table 1 Target Autoantigens in Pemphigus

\begin{tabular}{lll}
\hline \hline Subtypes & \multicolumn{1}{c}{ Antigens } & MW \\
\hline $\begin{array}{l}\text { Pemphigus vulgaris } \\
\text { Mucosal dominant type }\end{array}$ & desmoglein3 & $130 \mathrm{kD}$ \\
Mucocutaneous type & desmoglein3 & $130 \mathrm{kD}$ \\
& desmoglein1 & $160 \mathrm{kD}$ \\
Pemphigus foliaceus & desmoglein 1 & $160 \mathrm{kD}$ \\
Paraneoplastic pemphigus & desmoglein3 & $130 \mathrm{kD}$ \\
& desmoglein1 & $160 \mathrm{kD}$ \\
& plectin/HD1 & $500 \mathrm{kD}$ \\
& desmoplakinI & $250 \mathrm{kD}$ \\
& desmoplakinI & $210 \mathrm{kD}$ \\
& BPAG1 & $230 \mathrm{kD}$ \\
& envoplakin & $210 \mathrm{kD}$ \\
& periplakin & $190 \mathrm{kD}$ \\
& $?$ & $170 \mathrm{kD}$ \\
\hline
\end{tabular}

* Pemphigus vegetans is a variant of pemphigus vulgaris. Pempigus erythematosus and Brazilian pemphigus (fogo selvagem) is a variant of pemphigus foliaceus.

cell adhesion of keratinocytes with resultant blister formation. Immunochemical characterization of pemphigus antigens by immunoprecipitation or immunoblotting with extracts from cultured keratinocytes or epidermis has demonstrated that the pemphigus vulgaris and foliaceus antigens are $130 \mathrm{kDa}$ and $160 \mathrm{kDa}$ transmembrane glycoproteins, respectively (Table 1). ${ }^{4-8}$ Molecular cloning of cDNA encoding pemphigus antigens indicates that both these molecules are members of the cadherin supergene family. ${ }^{9,10}$ Pemphigus foliaceus and vulgaris antigens are termed desmoglein1 (Dsg1) and desmoglein 3 (Dsg3), respectively. Thus, pemphigus was discovered to be an anti-cadherin autoimmune disease. The basic pathophysiology of pemphigus is that autoantibodies inhibit the adhesive function of desmogleins and lead to the loss of cell-cell adhesion of keratinocytes with resultant blister formation.

Compelling evidence has accumulated that $\operatorname{IgG}$ autoantibodies against Dsg1 and Dsg3 are pathogenic and play a primary role in inducing the blister formation in pemphigus. IgG that is affinity-purified from pemphigus vulgaris sera on the extracellular domain of Dsg3 can cause suprabasilar acantholysis, the typical histologic finding of pemphigus vulgaris, when injected into neonatal mice. ${ }^{11}$ Furthermore, when anti-Dsg3 $\mathrm{IgG}$ is immunoadsorbed with the extracellular domains of Dsg3 from pemphigus vulgaris sera, those sera no longer have the pathogenic activity to cause blisters in neonatal mice. ${ }^{12}$ Similarly, immunoadsorption of pemphigus foliaceus sera with the extracellular domains of Dsg1 eliminates the pathogenic activity of those sera and anti-Dsg1 IgG bound on the column can cause superficial blisters in neonatal mice. ${ }^{13}$

\section{Desmoglein Compensation Theory as an Explanation for Localization of Blisters}

The disruption of desmoglein-dependent cell adhesion by autoantibodies is the basic pathophysiology in blister formation of pemphigus, but the clinical spectrum of pemphigus is more complex than this. Splits associated with pemphigus foliaceus occur in the superficial layer of the epidermis, while those of pemphigus vulgaris occur deep in the epidermis. Oral erosions are developed in patients with pemphigus vulgaris, but not in patients with pemphigus foliaceus. Some patients with pemphigus vulgaris have only oral involvement, but others have extensive lesions on both skin and mucous membranes. These complex clinical features of pemphigus are explained logically by the desmoglein compensation theory: i.e. Dsg1 and Dsg3 compensates for their adhesive function when they are coexpressed in the same cell (Fig. 1). ${ }^{14-16}$

When pemphigus vulgaris is divided into two subgroups, the mucosal dominant type which is demonstrated by mucosal lesions with minimal skin involvement and the mucocutaneous type which is demonstrated by extensive skin blisters and erosions in addition to mucosal involvement, each subtype of pemphigus has its own anti-desmoglein antibody profile (Table 1). Patients with pemphigus foliaceus have only anti-Dsg1 IgG autoantibodies. Patients with the mucosal dominant type of pemphigus vulgaris have only anti-Dsg3 IgG autoantibodies, while the mucocutaneous type of pemphigus vulgaris has both anti-Dsg3 and anti-Dsg1 IgG autoantibodies. ${ }^{17}$ The intraepithelial expression pattern of Dsg1 and Dsg3 are different between skin and the mucous membranes. In the skin, Dsg1 is expressed throughout the epidermis, but more intensely in the superficial layers, while Dsg3 is expressed in the lower part of the epidermis, mainly in the basal and parabasal layers. ${ }^{18}$ In contrast, in the mucous membranes, Dsg1 and Dsg3 are expressed throughout the squamous mucosal epithelia, but Dsg1 is expressed at a much lower level than Dsg3. ${ }^{14}$

When sera contain only anti-Dsg1 IgG which interferes with the function of Dsg1, those sera cause blisters only in the superficial epidermis because that is the only area in which Dsg1 is present without coexpression of Dsg3 (Fig. 1A). In the unaffected deep epidermis, the presence of Dsg3 compensates for the loss of function of Dsg1. Although the anti-Dsg1 IgG binds to the mucosa, no blisters are formed because of the coexpression of Dsg3. Thus, sera containing only antiDsg1 IgG cause superficial blisters in the skin without mucosal involvement as seen in patients with pemphigus foliaceus. When sera contain only anti-Dsg3 IgG, those sera do not effectively cause blisters in the skin because the co-expressed Dsg1 compensates for 
A. Pemphigus foliaceus

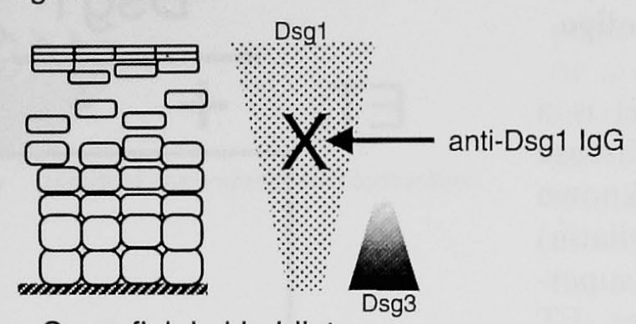

Superficial skin blisters

B. Mucosal dominant pemphigus vulgaris

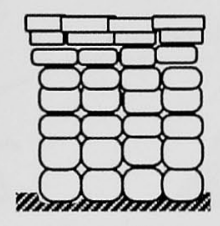

No or limited skin lesion

C. Mucocutaneous pemphigus vulgaris

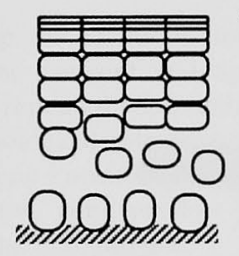

Deep skin blisters

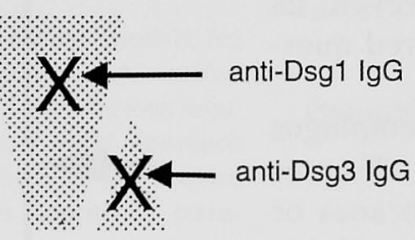

anti-Dsg3 IgG

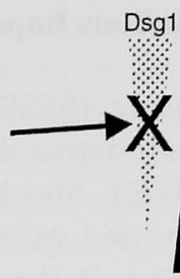

Dsg3
No mucosal lesion
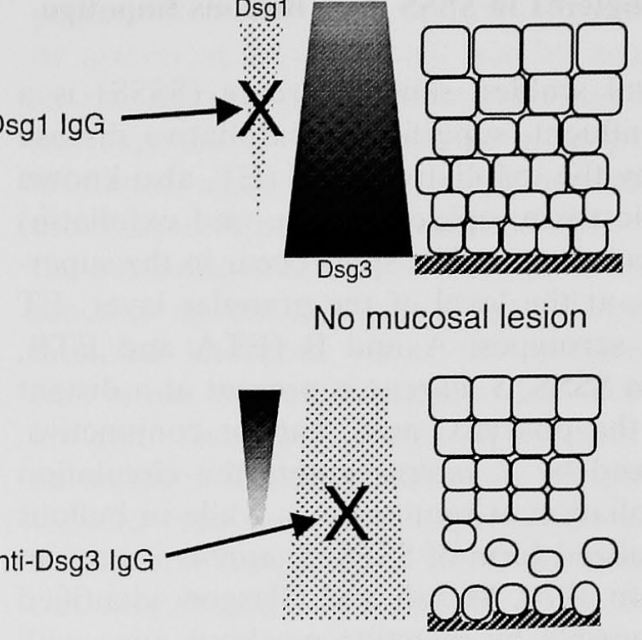

Mucosal erosions
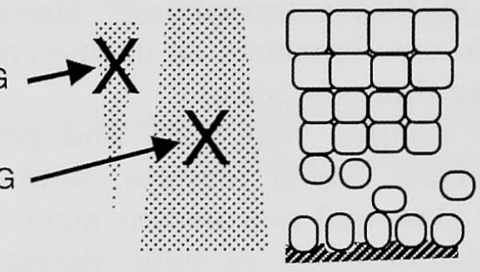

Mucosal erosions

Mucous membrane

Fig. 1 Explanation of the sites of blisters in pemphigus by the desmoglein compensation theory. The triangles represent the distribution of desmoglein1 (Dsg1) and desmoglein3 (Dsg3) in the skin and mucous membranes. Pemphigus foliaceus sera contain only anti-Dsg1 IgG and cause superficial blisters in the skin because Dsg3 functionally compensates for the impaired Dsg1 in the lower part of the epidermis, while these sera do not cause blisters in the mucous membrane because cell-cell adhesion is mainly mediated by Dsg3 (A). Sera containing only antiDsg3 IgG cause no or only limited blisters in the skin because Dsg1 compensates for the loss of the Dsg3-mediated adhesion, whereas those sera induce separation in the mucous membrane where the low expression of Dsg1 will not compensate for the loss of the Dsg3-mediated adhesion (B). When sera contain both anti-Dsg1 and anti-Dsg3 IgG, the function of both Dsgs are compromised and blisters occur both in the skin and mucous membranes $(C)$.

the impaired function of Dsg3, resulting in no or only limited skin blisters (Fig. 1B). In contrast, in mucous membranes, Dsg1 cannot compensate for the impaired Dsg3 function because of its low expression. Therefore, sera containing only anti-Dsg3 IgG cause oral erosions without apparent skin involvement as seen in patients with the mucosal dominant type of pemphigus vulgaris. When sera contain both anti-Dsg1 and anti-Dsg3 IgG, they interfere with the function of both Dsg1 and Dsg3, resulting in extensive blisters and erosions in the skin as well as the mucous membranes, as seen in patients with the mucocutaneous type of pemphigus vulgaris (Fig. 1C). It is not clear why splits occur just above the basal layer instead of the whole keratinocyte falling apart, it is however speculated that the cell-cell adhesion between the basal and parabasal layers might be weaker than the other parts of the epidermis because there are fewer desmosomes. In addition, the lower part of the epidermis might offer better access for autoantibodies penetrating from the dermis.

In pregnant women with pemphigus, autoantibodies cross the placenta and bind to the fetal epidermis. Neonates develop blisters from mothers with pemphigus vulgaris, but very rarely from mothers with pemphigus foliaceus. The reason for this paradoxical observation is also explained by the desmoglein compensation theory. ${ }^{19}$ The distribution of Dsg3 in the neonatal epidermis is unlike that in the adult epidermis and is found on the surface of keratinocytes throughout the epidermis, which is more like the distribution in mucous membranes because the neonatal skin was in amnio. Therefore, pemphigus foliaceus sera containing only anti-Dsg1 IgG cannot induce blisters in neonatal skin. 


\section{Staphylococcus Aureus Exfoliative Toxin Directly Digests Desmoglein1 in SSSS And Bullous Impetigo}

Staphylococcal scalded skin syndrome (SSSS) is a generalized, confluent, superficially exfoliative disease that is caused by the exfoliative toxin (ET, also known as epidermolytic toxin, epidermolysin, and exfoliatin) of Staphylococcus aureus. The splits occur in the superficial epidermis, at the level of the granular layer. ET has two major serotypes: A and B (ETA and ETB, respectively). In SSSS, $S$. aureus is present at a distant focus, such as the pharynx, nose, ear, or conjunctiva, and ET produced by $S$. aureus enters the circulation and causes exfoliation at remote sites, while in bullous impetigo, a localized form of SSSS, S. aureus is present in the lesions. In 1970, Melish and Glasgow identified the toxin responsible by injecting newborn mice with broth cultures of $S$. aureus isolated from patients with SSSS, resulting in blister formation. ${ }^{20}$ However, its mode of action in vivo remained an unanswered question for more than three decades.

One day, we realized that SSSS and pemphigus foliaceus share many similar features. Both diseases involve only the skin, and not mucous membranes or other tissues. The histology of both diseases shows superficial epidermal separation where Dsg3 is not coexpressed. Pathology textbooks actually say that the histology of both diseases is indistinguishable. When IgG from PF is injected into neonatal mice, the mice develop blisters. When ET is injected into neonatal mice, the mice also develop blisters. The histology in both mice is essentially identical. These similarities caused us to consider whether the molecular mechanism of blister formation in SSSS was actually similar to that in PF, and we started to investigate the fate of Dsg1 after ET treatment. ${ }^{21}$

We first stained Dsg1 and Dsg3 in mouse skin one hour after injecting ETA into neonatal mice. ETA caused substantial changes in Dsg1 staining, which was much less intense on keratinocyte cell surfaces, while there was no apparent change in Dsg3 staining. We next examined whether Dsg1 is degraded after ETA treatment. We performed an immunoblot analysis for Dsg1, Dsg3, and E-cadherin on extracts of skin from the mice that developed blisters. The 160-kDa Dsg1 was degraded into a peptide of approximately $113 \mathrm{kDa}$, while there was no degradation of Dsg3 or E-cadherin. To demonstrate direct proteolysis of the extracellular domain of Dsg1 by ETA, we incubated a soluble recombinant form of the extracellular domains of Dsg1 and Dsg3 with ETA in vitro (Fig. 2). ETA cleaved the recombinant mouse and human Dsg1 in a dosedependent fashion, while ETA did not cleave Dsg3 at all. Combined, these findings indicate that ETA specifically recognizes and cleaves the extracellular domain of

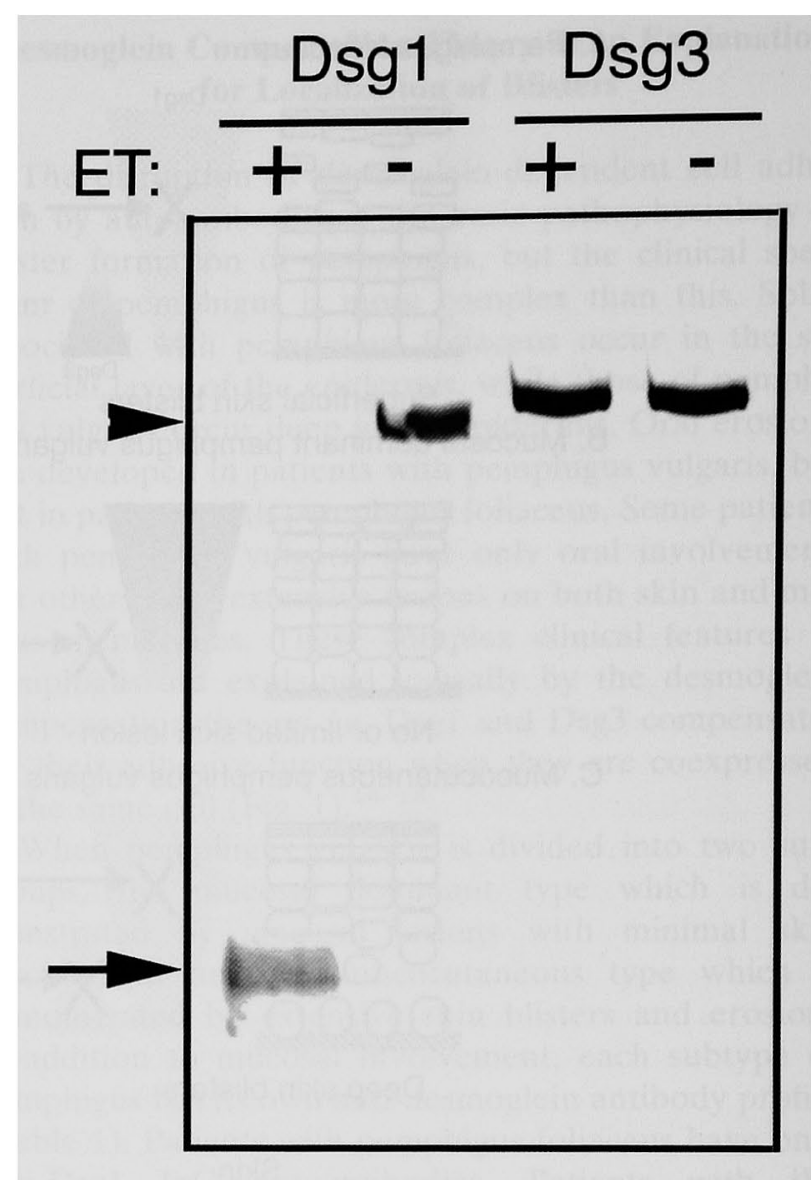

Fig. 2 In vitro digestion of the recombinant extracellular domains of human Dsg1 by exfoliative toxin. Dsg1 and Dsg3 extracellular domains produced in baculovirus were incubated with or without exfoliative toxin A (ET) and subjected to immunoblotting to visualize Dsg1 and Dsg3. The toxin cleaved the extracellular domain of Dsg1, but not that of Dsg3. The arrowhead and arrow indicate intact recombinant Dsg1 and the cleaved product, respectively.

both mouse and human Dsg1. Subsequently, we also demonstrated that ETB, another major serotype of ET, specifically cleaved Dsg1 in a manner identical to ETA. $^{22}$

These findings provide an important framework to understand the molecular mechanism of blister formation in these diseases, as well as cell-to-cell adhesion of keratinocytes in the epidermis. By bringing dermatological tools to bear on our observations we were thus able to clarify of the molecular mechanism of an exfoliative toxin, which had remained unsolved for three decades after its identification.

\section{A Novel Autoimmune Mouse Model Using the Autoantigen Knockout Mouse}

To investigate the pathophysiological mechanisms and to develop therapeutic strategies, animal disease models have been playing important roles in the study 


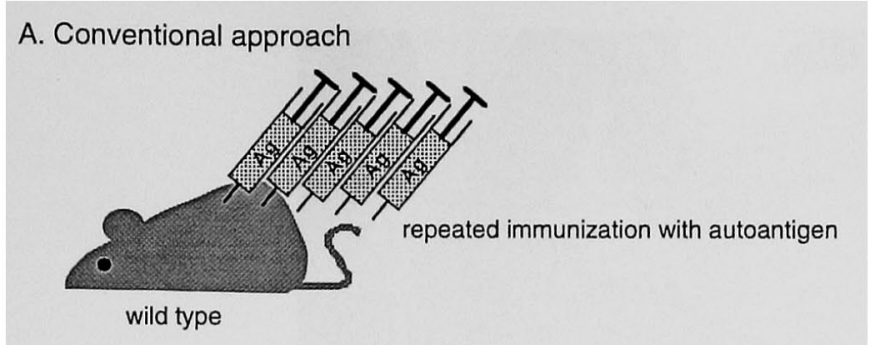

B. Novel approach using autoantigen knockout mouse

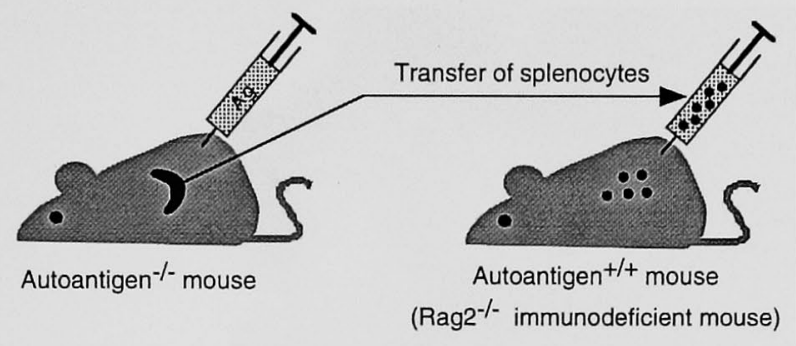

Fig. 3 Methods to develop an active disease mouse models for autoimmune diseases. In the conventional approach (A), various strains of wild type mice are repeatedly immunized with various adjuvants to break their immunological tolerance. In a novel approach (B), splenocytes of autoantigen knockout mice which do not acquire tolerance against the defective gene product are adoptively transferred to mice that express the autoantigen.

of various conditions including autoimmune diseases. To examine the cellular mechanisms of autoantibody production in pemphigus, specimens from patients are naturally the best clinical material, but it is not easy to obtain sufficient amounts with proper controls. To overcome this problem an active disease model is required. The conventional approach to develop an autoimmune mouse model is forced immunization of autoantigens in various strains of mice (Fig. 3A). However, this approach is empirical and immune responses are largely dependent on the strains of mice or types of adjuvant used. Furthermore, any autoimmune reaction in those mice may be transient, unlike that found in patients, and the immune system is systemically stimulated.

The major difficulty in the development of an autoimmune reaction in mice is because of the self-tolerance factor inherent in homeostasis, which prevents the immune system from reacting destructively against selfcomponents. When lymphocytes are exposed to selfcomponents during the development of the immune system, auto-reacting lymphocytes are eliminated or inactivated. We have taken a novel approach to overcome this problem. Because self-tolerance is a technical barrier to the development of autoimmune mouse models we thought of generating a condition where self-tolerance is not established in an antigen-specific way. If it were possible to remove the antigen during the development of the immune system, or if were not present from the start, tolerance against the removed or absent molecule would not be acquired (Fig. 3B). In the autoantigen knockout mouse, lymphocytes are not exposed to the defective gene product, and selftolerance against that particular autoantigen is not established. Upon immunization with the autoantigen, the autoantigen knockout mice should elicit an immune reaction against the autoantigen. However, in the immunized knockout mice, the antigen-antibody reaction is not expected because the mice lack the target antigen. Therefore, lymphocytes from the immunized autoantigen knockout mice are adoptively transferred to wild type mice that express the autoantigen. The transferred lymphocytes from the autoantigen knockout mice should be persistently stimulated by the endogenous autoantigen in the recipient mice and should therefore produce antibodies against the autoantigen with resultant phenotypes of the human disease.

\section{Pemphigus Mouse Model with Persistent Pathogenic Antibody Production}

To develop an active disease mouse model for pemphigus vulgaris, we took this novel approach of using autoantigen knockout mice, in the case of pemphigus vulgaris, Dsg3 $3^{-/-}$mice. ${ }^{23}$ When we immunized Dsg3 $3^{-1-}$ mice with mouse recombinant Dsg3, anti-Dsg3 IgG was indeed produced. These sera were able to bind to the cell surfaces of living cultured mouse keratinocytes, indicating that the anti-Dsg3 IgG produced in Dsg $3^{-1-}$ mice is capable of binding to the native Dsg3 on living keratinocytes. In contrast, sera from Dsg $3^{+1-}$ littermates or wild type mice failed to bind to the surface of living keratinocytes. These findings confirmed that Dsg $3^{-1-}$ mice and mice expressing Dsg3 have a clear difference in their ability to produce anti-Dsg3 IgG that can bind to the native Dsg3. ${ }^{24}$

Despite the production of anti-Dsg3 IgG, no autoimmune reaction is expected in the immunized Dsg $3^{-/-}$ mice because they lack the target antigen. To allow the anti-Dsg3 IgG to be exposed to the antigen, we isolated splenocytes from the immunized Dsg $3^{-1-}$ mice and transferred them into Rag-2-/- immunodeficient mice that do express Dsg3. Rag-2-1- mice have no mature $T$ or $B$ cells due to the inability to rearrange $T$ cell receptors or immunoglobulin genes and thus are unable to produce antibodies or reject the transferred splenocytes. Circulating anti-Dsg3 IgG was detected in the sera of recipient Rag- $2^{-1-}$ mice as early as day 4 after the transfer of $\mathrm{Dsg}^{-/-}$splenocytes, and its titer increased rapidly without further boosting by recombinant Dsg3, and reached a plateau around day 21. The circulating anti-Dsg3 IgG was detected for as long as 6 


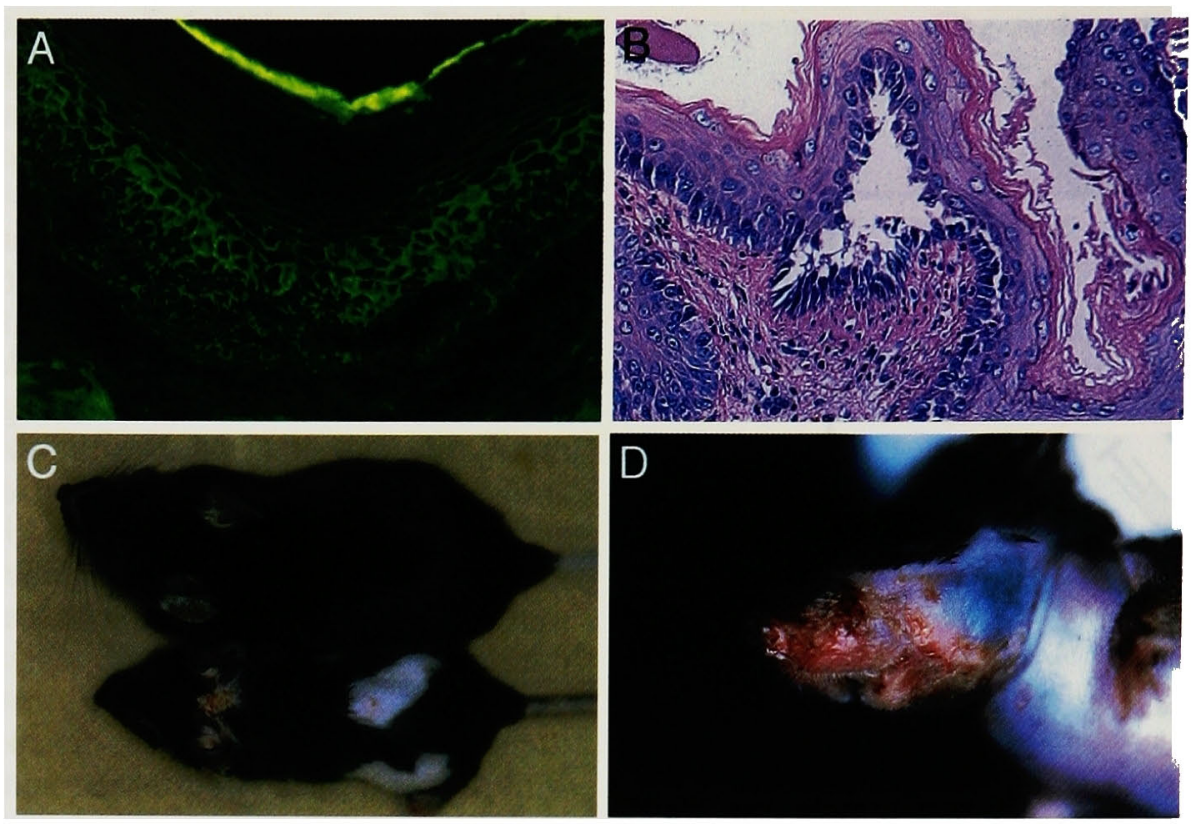

Fig. 4 Phenotype of active disease model mice for pemphigus vulgaris. Recipient mice with Dsg $3^{-/-}$splenocytes show in vivo mouse IgG deposition on keratinocyte cell surfaces (A) and suprabasilar acantholysis (B) just as in patients with pemphigus vulgaris. The recipient mice with Dsg $3^{-1-}$ splenocytes (the lower mouse in C) are smaller than control mice with Dsg $3^{+/-}$splenocytes (the upper mouse in C) because oral erosions inhibit food intake. Some recipient mice with Dsg ${ }^{-1-}$ splenocytes show crusted erosions around the snout and cheeks, where mice normally scratch (D).

months or more. No significant reactivity against Dsg1, another desmosomal cadherin targeted in pemphigus foliaceus, was observed in these recipient mice during this period. In contrast, no circulating anti-Dsg3 IgG was detected in $\mathrm{Rag}-2^{-/-}$mice given $\mathrm{Dsg} 3^{+/-}$splenocytes. The persistent anti-Dsg3 IgG production indicates that endogenous Dsg3 in the recipient mice stimulated the transferred Dsg3-specific lymphocytes from the immunized $\mathrm{Dsg} 3^{-/-}$mice in vivo.

In recipient mice with Dsg $3^{-/}$splenocytes, in vivo $\mathrm{IgG}$ deposition was found on keratinocyte cell surfaces in stratified squamous epithelia, including the skin and oral and esophageal mucous membranes, just as seen in patients with pemphigus vulgaris (Fig. 4A). In these mice, no IgG deposition was found in other tissues, including heart, lung, liver, kidney, stomach, and small and large intestines. These IgG binding sites correspond to the known tissue distribution of Dsg3. Histological examination of the recipient mice revealed an intraepithelial loss of cell-cell adhesion just above the basal layers, i.e., suprabasilar acantholysis, in the buccal mucosa, hard palate, oropharyngeal areas, and the upper part of the esophagus, just as in human patients (Fig. 4B). These oral erosions likely inhibited food intake, resulting in the weight loss (Fig. 4C). Some of the recipient mice developed crusted erosions on the skin around the snout, an area that is normally traumatized by scratching (Fig. 4D). Close histological examination revealed that the recipient mice with Dsg3 $3^{-1-}$ splenocytes also exhibited eosinophilic spongiosis which is often found in patients with early lesions. ${ }^{25} \mathrm{We}$ also observed patchy hair loss in the recipient mice with Dsg $3^{-1-}$ splenocytes. This hair loss phenotype also persisted for over 6 months. Skin biopsy showed intense IgG deposition on the cell surface of keratinocytes surrounding the telogen hair club. Cleft formation was observed between the cells surrounding the telogen club and the basal layer of the outer root sheath epithelium. In contrast, no phenotypic or pathological changes were observed in recipient mice with Dsg $3^{+/-}$ splenocytes. These results indicate that the Rag-2-/recipient mice given immunized Dsg $3^{-/-}$splenocytes developed clinical, histologic and immunopathologic phenotypes similar to those of patients with pemphigus vulgaris. ${ }^{24}$

A major hurdle in developing animal models of autoimmune diseases has been overcoming the selftolerance component of the homeostatic system. We circumvented this problem by immunizing autoantigen knockout mice with the autoantigen, then transferring their splenocytes to Rag-2-/- mice that expressed the autoantigen. Although this model does not reflect the actual triggers of autoimmune diseases, it does provide a means to investigate the roles of $\mathrm{T}$ and $\mathrm{B}$ lymphocytes in perpetuating autoantibody production in the autoimmune response. ${ }^{26} \mathrm{We}$ are also attempting to isolate 
a panel of monoclonal anti-Dsg3 IgG antibodies from pemphigus model mice, and investigate their pathogenic strength to clarify what factor(s) determines the severity of the disease. In addition, this active animal model should be beneficial for evaluating various therapeutic strategies that could modulate the autoimmune response. Finally, our approach is widely applicable to various antibody-mediated and $\mathrm{T}$ cell-mediated autoimmune diseases, unless the relevant autoantigen knockout mice are embryonicaly lethal or show gross abnormalities in their immune systems.

Acknowledgements: I acknowledge a deep debt of gratitude to Dr. Takeji Nishikawa for his generous support and guidance and Dr. John R. Stanley for his collaboration and stimulating discussion throughout the course of the studies mentioned in this manuscript. The pemphigus mouse model was developed with collaboration with Dr. Shigeo Koyasu. The works mentioned in this article were supported by Keio Gijuku Academic Development Funds, Health Sciences Research Grants for Research on Specific Diseases from Ministry of Health, Labour and Welfare, and a Grant-in-Aid for Scientific Research from the Ministry of Education, Culture, Sports, Science and Technology of Japan.

\section{References}

1. Stanley JR: Pemphigus. In: Freedberg IM, Eisen AZ, Wolff K, et al, eds, Dermatology in General Medicine, 4th ed., New York, McGraw-Hill, 1998; 654-666

2. Anhalt GJ, Kim S, Stanley JR, Korman NJ, Jabs DA, Kory M, Izumi H, Ratrie H, Mutasim D, Ariss AL et al: Paraneoplastic pemphigus. An autoimmune mucocutaneous disease associated with neoplasia. N Engl J Med 1990; 323: 1729-1735

3. Beutner EH, Jordon RE: Demonstration of skin antibodies in sera of pemphigus vulgaris patients by indirect immunofluorescent staining. Proc Soc Exp Biol Med 1964; 117: 505-510

4. Stanley JR, Yaar M, Hawley NP, Katz SI: Pemphigus antibodies identify a cell surface glycoprotein synthesized by human and mouse keratinocytes. J Clin Invest 1982; 70: 281-288

5. Stanley JR, Koulu L, Thivolet C: Distinction between epidermal antigens binding pemphigus vulgaris and pemphigus foliaceus autoantibodies. J Clin Invest 1984; 74: 313-320

6. Eyre RW, Stanley JR: Human autoantibodies against a desmosomal protein complex with a calcium-sensitive epitope are characteristic of pemphigus foliaceus patients. J Exp Med 1987; 165: 1719-1724

7. Eyre RW, Stanley JR: Identification of pemphigus vulgaris antigen extracted from normal human epidermis and comparison with pemphigus foliaceus antigen. $\mathrm{J}$ Clin Invest 1988; 81: 807812

8. Hashimoto T, Ogawa MM, Konohana A, Nishikawa T: Detection of pemphigus vulgaris and pemphigus foliaceus antigens by immunoblot analysis using different antigen sources. J Invest Dermatol 1990; 94: 327-331

9. Koch PJ, Walsh MJ, Schmelz M, Goldschmidt MD, Zimbelmann $R$, Franke WW: Identification of desmoglein, a constitutive desmosomal glycoprotein, as a member of the cadherin family of cell adhesion molecules. Eur J Cell Biol 1990; 53: 1-12

10. Amagai M, Klaus-Kovtun V, Stanley JR: Autoantibodies against a novel epithelial cadherin in pemphigus vulgaris, a disease of cell adhesion. Cell 1991; 67: 869-877

11. Amagai M, Karpati S, Prussick R, Klaus-Kovtun V, Stanley JR: Autoantibodies against the amino-terminal cadherin-like binding domain of pemphigus vulgaris antigen are pathogenic. J Clin Invest 1992; 90: 919-926

12. Amagai M, Hashimoto T, Shimizu N, Nishikawa T: Absorption of pathogenic autoantibodies by the extracellular domain of pemphigus vulgaris antigen (Dsg3) produced by baculovirus. $\mathbf{J}$ Clin Invest 1994; 94: 59-67

13. Amagai $M$, Hashimoto $T$, Green $K J$, Shimizu N, Nishikawa $T$ : Antigen-specific immunoadsorption of pathogenic autoantibodies in pemphigus foliaceus. J Invest Dermatol 1995; 104: 895901

14. Shirakata Y, Amagai M, Hanakawa Y, Nishikawa T, Hashimoto $\mathrm{K}$ : Lack of mucosal involvement in pemphigus foliaceus may be due to low expression of desmoglein1. J Invest Dermatol 1998; 110: $76-78$

15. Mahoney MG, Wang Z, Rothenberger KL, Koch PJ, Amagai M, Stanley JR: Explanation for the clinical and microscopic localization of lesions in pemphigus foliaceus and vulgaris. J Clin Invest 1999; 103: 461-468

16. Amagai M: Autoimmunity against desmosomal cadherins in pemphigus. J Dermatol Sci 1999; 20: 92-102

17. Amagai $M$, Tsunoda $K$, Zillikens $D$, Nagai $T$, Nishikawa $T$ : The clinical phenotype of pemphigus is defined by the antidesmoglein autoantibody profile. J Am Acad Dermatol 1999; 40: $167-170$

18. Amagai M, Koch PJ, Nishikawa T, Stanley JR: Pemphigus vulgaris antigen (Desmoglein3) is localized in the lower epidermis, the site of blister formation in patients. J Invest Dermatol 1996; 106: $351-355$

19. Wu H, Wang ZH, Yan A, Lyle S, Fakharzadeh S, Wahl JK, Wheelock MJ, Ishikawa $\mathrm{H}$, Uitto J, Amagai M et al: Protection against pemphigus foliaceus by desmoglein 3 in neonates. $\mathrm{N}$ Engl J Med 2000; 343: 31-35

20. Melish ME, Glasgow LA: The staphylococcal scalded skin syndrome: Development of an experimental model. N Engl J Med 1970; 282: 1114-1119

21. Amagai M, Matsuyoshi N, Wang ZH, Andl C, Stanley JR: Toxin in bullous impetigo and staphylococcal scalded skin syndrome targets desmoglein1. Nature Medicine 2000; 6: 1275-1277

22. Amagai M, Yamaguchi T, Hanakawa $Y$, Nishifuji K, Sugai M, Stanley JR: Staphylococcal Exfoliative Toxin B Specifically Cleaves Desmoglein1. J Invest Dermatol 2002; 118: 845-850

23. Koch PJ, Mahoney MG, Ishikawa H, Pulkkinen L, Uitto J, Shultz L, Murphy GF, Whitaker-Menezes D, Stanley JR: Targeted disruption of the pemphigus vulgaris antigen (desmoglein 3 ) gene in mice causes loss of keratinocyte cell adhesion with a phenotype similar to pemphigus vulgaris. J Cell Biol 1997; 137: 1091-1102

24. Amagai M, Tsunoda K, Suzuki H, Nishifuji K, Koyasu S, Nishikawa T: Use of autoantigen knockout mice to develop an active autoimmune disease model of pemphigus. J Clin Invest 2000; 105: 625-631

25. Ohyama M, Amagai M, Tsunoda K, Ota T, Koyasu S, Umezawa A, Hata J, Nishikawa T: Immunologic and histopathologic characterization of active disease mouse model for pemphigus vulgaris. J Invest Dermatol 2002; 118: 199-204

26. Tsunoda K, Ota T, Suzuki H, Ohyama M, Nagai T, Nishikawa T, Amagai M, Koyasu S: Pathogenic autoantibody production requires loss of tolerance against desmoglein 3 in both $T$ and $B$ cells in experimental pemphigus vulgaris. Eur J Immunol 2002; 32: $627-633$ 